\title{
Esquisse d'une sociologie des réseaux festivaliers. Le cas du Festival TransAmériques de Montréal
}

\author{
SYLVAIN SCHRYBURT
}

\begin{abstract}
Depuis le milieu des années 1970, on assiste à la multiplication du nombre de festivals et de diffuseurs saisonniers dont tout ou partie de la programmation est consacrée au théâtre étranger. Ces festivals et diffuseurs-qui se comptent aujourd'hui par centaines-sont principalement concentrés en Europe de l'Ouest et forment de vastes circuits informels que privilégient les artistes du théâtre québécois depuis les années 1980 lorsqu'ils tournent à l'extérieur de leurs frontières. Montréal appartient en propre à ces circuits internationaux depuis la fondation du Festival de Théâtre des Amériques (1985-2006) et celle de son successeur, le Festival TransAmériques (2007), dont l'étude sera au cœur du présent article. Inspirée de la sociologie des réseaux, cette étude explore les réseaux internationaux (personnels, de diffusion ou de coproduction) auxquels participent ce festival et ses représentants, de même que son rôle de médiateur entre l'ici et l'ailleurs.
\end{abstract}

Since the mid 1970s, there has been a considerable rise in the number of festivals and seasonal venues whose partial or complete programing is dedicated to international theatre. These festivals and seasonal venues-which today number in the hundreds-are mostly located in Western Europe and form vast yet informal circuits followed by Quebec theatre artists when touring abroad since the 1980s. Montreal is structurally part of such circuits since the establishment of the Festival de Théâtre des Amériques (1985-2006) and its successor the Festival TransAmériques (2007). Borrowing its theoretical framework from network sociology this article explores the international networks (personnel, touring, and coproduction) in which the Festival TransAmériques and its representatives participate as well as the festival's role as a mediator between here and abroad.

Alors que des artistes de théâtre du Québec se produisent à l'étranger depuis au moins la fin du XIX ${ }^{\mathrm{e}}$ siècle, ce n'est qu'avec les années I980 que le phénomène prend une ampleur sans précédent et qu'il perd du même coup son caractère d'exception. À partir de cette décennie, les tournées internationales de compagnies théâtrales québécoises se font non seulement plus nombreuses, elles deviennent aussi plus longues et géographiquement étendues. ${ }^{I}$ Parmi les quelque cinquante-quatre spectacles québécois présentés à l'étranger durant les années I980, qu'il suffise ici d'en rappeler trois qui sont demeurés emblématiques de la période et qui ont connu, en leur temps, un rayonnement international hors norme : Le Rail de Gilles Maheu (Carbone I4, I984), La Trilogie des dragons de Robert Lepage (Théâtre Repère, 1987) et Oulipo show de Denis Marleau (UBU, 1988). ${ }^{2}$ 
Ces metteurs en scène, mais aussi Marie Brassard, Wajdi Mouawad et plusieurs autres, ont pu bénéficier des vastes circuits internationaux de tournées qui se sont développés depuis le milieu des années I970, tout particulièrement en Europe de l'Ouest. Ces circuits sont pour l'essentiel composés de festivals et de diffuseurs saisonniers dont tout ou partie du mandat consiste à présenter au public de leur ville respective une sélection de spectacles de théâtre venus d'ailleurs, y compris du Québec. Ce sont dans de tels espaces que circule le théâtre québécois de pointe, là où il est aujourd'hui programmé aux côtés de quelques-unes des principales têtes d'affiche de la danse et du théâtre contemporains, soit des metteurs en scène ou des chorégraphes comme Romeo Castellucci, Boris Charmatz, Anne Teresa De Keersmaeker, Jan Fabre, Jan Lauwers, Thomas Ostermeier ou Joël Pommerat, des artistes dont on a pu voir le travail à Montréal.

En abordant la question de la circulation internationale du théâtre québécois, mon point de vue ne sera pourtant pas celui des artistes ou des œuvres, comme il serait d'usage, mais bien celui des festivals et diffuseurs saisonniers qui les accueillent. Je m’intéresserai surtout au plus important festival de théâtre basé au Québec, le Festival TransAmériques de Montréal (FTA, 2007) que dirigeait jusqu'à tout récemment sa fondatrice Marie-Hélène Falcon. ${ }^{3}$ Pour mémoire, rappelons que ce festival est le prolongement d'un festival plus ancien, le Festival de Théâtre des Amériques (FTA, 1985-2006), un évènement biannuel aussi fondé et dirigé par Falcon, et qui programmait une sélection du théâtre contemporain d'ici et d'ailleurs. ${ }^{4}$ Depuis sa refonte en TransAmériques, l'offre du FTA comprend entre 2I et 3I spectacles 5 que l'on peut envisager selon deux axes : celui de la discipline artistique et celui de l'origine géographique des spectacles. Au FTA, la programmation annuelle est ainsi composée à parts égales de danse et de théâtre contemporain. ${ }^{6}$ De même, son offre en danse et en théâtre provient pour moitié des scènes nationales (principalement québécoises, pour ne pas dire montréalaises) et pour moitié des scènes internationales (avec une dominance de l'Europe de l'Ouest).

Dans le cadre de cet article, l'enquête sera pour l'essentiel limitée à la version TransAmériques du festival dont la présence internationale s'est accrue considérablement en 2007, lorsqu'il est devenu annuel et pluridisciplinaire. Plus particulièrement, il s'agira d'interroger les réseaux de diffusion et de coproduction dans lesquels ce festival est actif, réseaux qui font de lui un joueur international de plein droit, mais aussi un allié pour les artistes québécois qu'il programme. Plus modestement, et à travers ce cas d'espèce, il s'agira aussi de penser les bases méthodologiques d'une analyse sociologique des réseaux festivaliers qui reste encore à développer.

\section{Saison du diffuseur, édition du festival}

Avant de poursuivre, qu'il me soit permis une remarque préliminaire. Elle concerne l'abandon quasi complet en ces pages de la catégorie des diffuseurs saisonniers, ${ }^{7}$ un ensemble hétéroclite d'organismes gestionnaires de lieux de théâtre avec qui les festivals entretiennent de nombreuses alliances et dont l'étude, faute d'espace, devra être reportée à des travaux ultérieurs. Disons simplement de cette catégorie de diffuseurs qu'elle comprend des lieux influents comme le Brooklyn Academy of Music de New York (I859) ou le Théâtre de la Ville de Paris (1968), qui programment ou coproduisent une sélection des metteurs en scène et 
des chorégraphes étrangers les plus en vue aux côtés d'artistes nationaux connus - ou nonà l'extérieur de leurs frontières. Les diffuseurs saisonniers comprennent aussi des lieux pluridisciplinaires comme le Barbican de Londres (I982), le Harbourfront Center de Toronto (I99I) ou le Haus der Berliner Festspiele (200I). Ils incluent enfin des "Scènes Nationales ", des « Maisons de la culture " et autres théâtres "nationaux » ou " municipaux ", suivant les appellations officielles qu'on donne à de telles institutions en France, en Belgique, en Allemagne ou ailleurs. ${ }^{8}$ C'est dire la variété des statuts et des mandats (légaux ou artistiques), mais aussi la disparité des moyens financiers des diffuseurs internationaux en théâtre. Depuis un contexte national qui lui est propre, l'Usine C (1996) serait la principale contrepartie montréalaise de tels organismes.

Tout comme le festival dont il sera surtout question ici, ces diffuseurs sont actifs dans les réseaux internationaux d'accueil et de coproduction d'œuvres de théâtre, y compris québécoises. Leur nom figure d'ailleurs dans les itinéraires de tournées d'artistes comme Marie Brassard ou Robert Lepage, à côté du FTA notamment. Il existe cependant une distinction fondamentale entre festivals et diffuseurs saisonniers qui explique pourquoi l'étude des premiers s'avère aujourd'hui prioritaire sur celle des seconds. Cette distinction repose sur leur inscription temporelle respective. Alors que la programmation d'un diffuseur est étalée sur plusieurs mois, parfois même une année entière, et se pense en tant que saison, celle d'un festival fonctionne sur le mode de l'édition, soit une accumulation, sur un court laps de temps, de spectacles et autres évènements publics ou privés. ${ }^{9}$

Cette capacité à présenter une masse critique d'œuvres dans un temps condensé transforme un festival en "méta-évènement ", c'est-à-dire, pour reprendre la formule d'Henri Shoenmakers, en " évènement composé d'évènements singuliers " (27). ${ }^{\text {IO }}$ Contrairement à un diffuseur saisonnier, un festival est donc un évènement en soi, un espacetemps où convergent spectacles et artistes, mais aussi un certain nombre de "professionnels ", dont des directeurs artistiques impliqués dans la diffusion internationale des œuvres. Or, c'est justement sa capacité à attirer des diffuseurs étrangers, on le verra, qui confère à la formule festivalière un avantage décisif dans la constitution et l'attribution d'un capital symbolique spécifique au sein de cet espace supranational.

\section{Espaces internationaux, lieux de médiation}

On l'a dit, les circuits de diffusion internationale empruntés par les artistes du Québec et d'ailleurs sont notamment composés de festivals dont tout ou partie du mandat consiste à diffuser localement du théâtre étranger. À côté des plus anciens, tels que les Holland Festival d'Amsterdam (1947), Festival d'Avignon (1947) ou Festwochen de Vienne (I95I), plusieurs autres villes telles que Paris (Festival d'Automne, I972), Genève (La Bâtie, I977), Londres (LIFT, I98I), Rome (Romaeuropa, I986), Bruxelles (Kunstenfestivaldesarts, 1994), Berlin (Spielzeit'Europa, 2004-2OI2, Foreign Affairs, 2012) mais aussi Melbourne (I986), Leipzig (I99I), Moscou (I992), Belgrade (1992), Mons (2000), Rotterdam (2004) ou Tokyo (2009) ont vu se créer des festivals où l'on propose une sélection du théâtre mondial, la plupart du temps aux côtés d'artistes locaux reconnus - ou non-à l'extérieur de leurs frontières nationales. Depuis plusieurs années déjà, le Canada et le Québec font partie intégrante de ces réseaux festivaliers, grâce notamment au PuSh de Vancouver (2003), mais surtout au Festival TransAmériques. 
Inscrit localement et en même temps tourné vers l'ailleurs, le FTA agit tel un médiateur, un lieu de circulation et de passage entre le local (Montréal), le national (Québec/Canada) et l'international (principalement l'Europe continentale de l'Ouest). Vue de l'étranger, une invitation du FTA donne accès à un champ théâtral local, celui de Montréal, qui s'avère autrement difficile à percer tant les lieux d'accueil y sont, en définitive, relativement peu nombreux. ${ }^{I I}$ Aux yeux de son public montréalais, la programmation annuelle du FTA ouvre en retour une fenêtre sur l'ailleurs en proposant, sur un temps court, une sélection de la création théâtrale étrangère de pointe. À titre de diffuseur international de théâtre, l'action médiatrice du festival agit donc de l'étranger vers Montréal.

Elle peut aussi agir en sens inverse, on s'en doute, puisque le festival offre une tribune à quelques artistes québécois sélectionnés dans le volet canadien de sa programmation théâtrale (6 spectacles pour l'édition 20I3, dont I hors Québec). Dans le meilleur des cas, cette tribune pourra à terme mettre une œuvre (et un artiste) du Québec en contact avec des directeurs artistiques étrangers, ce qui peut lui valoir d'être inclus dans la programmation des festivals ou diffuseurs qu'ils représentent. Les exemples devenus emblématiques de cette action médiatrice du FTA demeurent à ce jour La Trilogie des Dragons de Robert Lepage (édition I987) et fimmy, créature de rêve de Marie Brassard (édition 200I), un spectacle sur lequel j'aurai l'occasion de revenir plus loin.

\section{La perspective des réseaux}

Qu'il agisse du local vers l'étranger ou de l'étranger vers le local, le rôle de médiateur d'un festival-son action internationale - suppose qu'il participe à tout un ensemble de réseaux interconnectés, réseaux dont la saisie globale - tâche évidemment impossible - tracerait les frontières mouvantes et poreuses d'espaces internationaux dont quelques-unes des dynamiques structurantes ont pu être étudiées ailleurs sous l'angle d'une sociologie du champ (Schryburt 2010, 2013; Ferguson).

Par réseau, j'entendrai ici une «structure formée des relations qui lient des acteurs sociaux $^{12}$ " (Marsden 2727). Ces " acteurs " sont pour l'essentiel des individus, bien que la notion puisse aussi inclure des institutions (Mesure et Savidan Ioro), une double acception dont je me prévaudrai volontiers. J'interrogerai d'abord la composition du public des professionnels $^{13}$ qui fréquentent un festival comme le FTA ainsi que les réseaux personnels dont ils témoignent. Ensuite seulement, j'aborderai la question depuis la perspective des acteurs institutionnels que sont les festivals et les diffuseurs saisonniers. Dans ce cadre élargi, j'explorerai les réseaux de diffusion et de coproduction qui tissent des liens tantôt faibles tantôt forts entre le Festival TransAmériques et ses pairs, principalement d'autres festivals et diffuseurs.

\section{Les réseaux d'acteurs sociaux}

La directrice artistique du FTA et ses mandataires voyagent à l'étranger à la recherche de spectacles à programmer dans leur propre évènement, mais aussi à la rencontre d'artistes et de démarches artistiques singulières. C'est qu'en leur qualité de " méta-évènement ", et grâce à l'attrait de leur temporalité condensée, les festivals étrangers sont pour les représentants du FTA des lieux de sociabilité privilégiés où nouer et entretenir des liens avec des artistes, des journalistes ou des professionnels de la scène en provenance de divers pays. À l'inverse, 
le FTA invite et reçoit chez lui, lors de son édition annuelle, des artistes, des journalistes et des professionnels d'ici ou d'ailleurs, lesquels constituent autant de publics stratégiques pour un festival international.

Ces publics, souvent à cause de leur travail, sont plus susceptibles que d'autres d'être des "festival participant» (i.e. des festivaliers) plutôt que de simples « spectateurs ». Cette distinction, proposée par Shoenmakers (30), fait du « festivalier » un spectateur qui a conscience de participer à un " méta-évènement ", un spectateur qui saisit le contexte festivalier plus large dans lequel s'inscrit l'évènement singulier auquel il assiste. Parmi tous les types de festivaliers qui fréquentent le FTA, celui des "professionnels de la scène " a ceci de particulier qu'il est, pour un festival, ce qui s'apparente le plus à un public de pairs.

Employée par le FTA comme par d'autres festivals, cette étiquette des "professionnels » recoupe en effet les directions artistiques de festivals et de diffuseurs saisonniers (nationaux ou étrangers), les agents d'artiste, les administrateurs de compagnies, les directeurs de centres d'auteurs ou, plus rarement, les fonctionnaires de la culture, soit des individus qui jouent un rôle décisionnel non directement artistique dans la production des œuvres et dans leur diffusion, notamment à l'étranger.

Le rayonnement du FTA, sa réputation comme sa sphère d'influence, tiennent donc en partie à sa capacité à attirer, d'une édition à l'autre, des médiateurs qui, comme lui, sont actifs dans la diffusion nationale et internationale. Le nombre et l'influence des "professionnels " qui fréquentent un festival comme le FTA offrent ainsi une mesure qualitative de l'intérêt que suscite sa programmation pour le public des pairs. C'est ce capital symbolique que fait jouer la direction du festival lorsqu'elle souligne la présence, lors de sa dernière édition, de "II8 professionnels de la scène en provenance de I7 pays " (Couturier, Io juin 2013). ${ }^{14}$ Outre le FTA, d'autres festivals publient des chiffres en empruntant des catégories similaires, mais jamais identiques, ce qui rend leur comparaison difficile, voire impossible. Citons en exemple le Festival d'Avignon et ses " 3500 professionnels du spectacle vivant " (Festival d'Avignon), le Carrefour international de théâtre de Québec et ses " 453 artistes et professionnels " (Charlebois, 2013) ou encore les «230 practioners, aficionados and industry professionals " présents au dernier PuSh International Performing Arts Festival de Vancouver (PuSh).

Ces chiffres, dont on ne connaît d'ailleurs rien du détail, ${ }^{15}$ témoignent des réseaux personnels que tissent entre eux les directeurs artistiques et autres décideurs impliqués dans la diffusion internationale du théâtre contemporain. Ils laissent entrevoir tout un monde de sociabilités, d'alliances, d'affinités, de luttes, de rapports interpersonnels ; en un mot, tout l'éventail des liens sociaux concrets qu'entretient une personne comme Marie-Hélène Falcon dans le cadre de ses fonctions de directrice artistique.

Or, parce qu'ils s'inscrivent dans des relations de travail, il importe de rappeler que ces réseaux professionnels existent, en dernière analyse, pour permettre ou faciliter la diffusion d'œuvres théâtrales. En ce sens, les réseaux professionnels s'accomplissent tout particulièrement lorsqu'ils se transforment en réseaux institutionnels, c'est-à-dire lorsque l'individu MarieHélène Falcon établit sa programmation annuelle et que ses choix engagent désormais l'institution qu'elle représente. Situons donc l'analyse à un autre niveau afin de penser cette fois les réseaux d'acteurs institutionnels qui lient le FTA à ses pairs, soit les festivals et diffuseurs saisonniers qui s'impliquent, comme lui, dans la diffusion et la coproduction de spectacles. 


\section{Les réseaux institutionnels de diffusion : des liens faibles}

Passer des réseaux professionnels de Falcon aux réseaux institutionnels du FTA suppose que l'on revoit d'abord la notion de « liens concrets » (Lacroix 2003:490) entre acteurs, liens qui sont la condition essentielle à la formation de réseaux. Que signifie un «lien concret » entre deux ou plusieurs institutions? Entre le FTA et l'un ou l'autre de ses pairs festivals ou diffuseurs saisonniers? Dans la mesure où tous ont pour mandat-même partiel-de programmer une sélection du théâtre contemporain, les points de contacts ou liens concrets entre le FTA et ses homologues étrangers seront les spectacles eux-mêmes qui, circulant d'une ville à l'autre, forment des réseaux de diffusion qui unissent le festival montréalais aux autres institutions qui les ont accueillis.

Prenons un exemple récent : la production par la Schaubühne d'Un ennemi du peuple d'Henrik Ibsen dans la mise en scène de Thomas Ostermeier. Créé au Festival d'Avignon en juillet 2OI2, ce spectacle a été présenté au Melbourne Festival puis au TNP à Lyon avant de connaître sa première nord-américaine au FTA, le 23 mai 20I3, soit quatre jours avant que le Carrefour International de Théâtre de Québec n'accueille à son tour la production. Et ainsi de suite pour le Hellenic Festival d'Athènes, le Istanbul Theatre Festival, le Théâtre de la Ville de Paris et les dix autres festivals et diffuseurs qui ont programmé-c'est aussi dire acheté-ce spectacle au moment d'écrire ces lignes.

Litinéraire de tournée d'Un ennemi du peuple donne ainsi à voir toute l'étendue géographique du réseau international de diffusion auquel participe le FTA pour avoir inclus ce spectacle dans son édition 20I3. Vu en diachronie, ce réseau aurait l'apparence suivante :

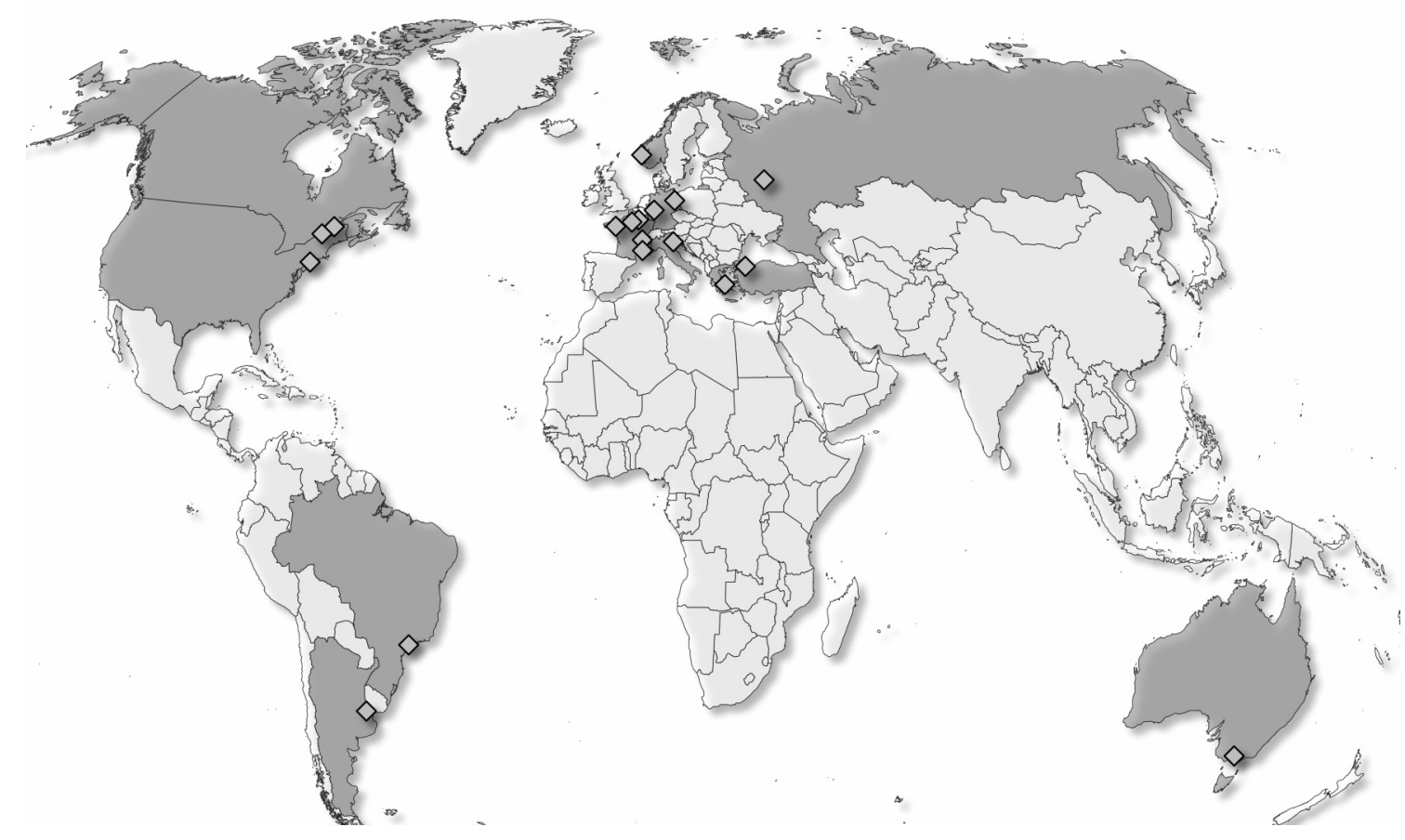

Réseau de diffusion d'Un ennemi de peuple (Thomas Ostermeier) Juillet 2012 - Août 2013 
Un réseau de diffusion comme celui-ci repose sur des liens faibles entre acteurs institutionnels. Ladjectif faible - ou lâche-, suppose ici des liens indirects entre institutions. Dans le cas des réseaux de diffusion, les liens entre festivals et diffuseurs saisonniers sont indirects car médiatisés par des spectacles. C'est en accueillant Un ennemi du peuple à Montréal que la directrice artistique du FTA inscrit de facto son festival dans le réseau de diffusion international constitué des festivals et autres diffuseurs qui ont, comme lui, choisi d'inscrire Ostermeier dans leur programmation. Il en résulte des points de contact ou liens concrets entre programmations, liens qui laissent entrevoir des communautés «électives " (Degenne et Forsé 2004 : 39). ${ }^{16}$ Dans l'espace qui nous intéresse, ces communautés se nouent autour des choix esthétiques partagés entre directions artistiques. Un réseau de diffusion comme celui d'Un ennemi du peuple dévoile ainsi un lien d'affinité esthétique que partage le FTA et dix-sept autres festivals ou diffuseurs.

Que signifie ce lien? Quelle en est la force? Peu de chose, si l'on songe qu'Un ennemi du peuple n'est qu'un des soixante-douze spectacles étrangers accueillis à Montréal par le FTA entre 2007 et 2013. C'est que, pris isolément, le réseau de diffusion en dit plus long sur la circulation d'un spectacle en particulier que sur les affinités entre les programmations des festivals et diffuseurs qui l'ont présenté. Pour que ces affinités apparaissent plus clairement, il convient d'augmenter le nombre de cas singuliers, c'est-à-dire de spectacles, et de lire le phénomène en diachronie. On obtient alors un indicateur plus précis de la densité des liens esthétiques entre festivals et diffuseurs.

Ce faisant, il apparaît que sur les soixante-douze spectacles étrangers présentés au FTA depuis 2007, soit sur sept éditions, vingt-deux l'ont aussi été au Théâtre de la Ville de Paris. Un peu moins du quart de la programmation étrangère globale du FTA est ainsi en partage avec ce diffuseur saisonnier français, une proportion qui grimpe à plus de deux sur cinq si l'on isole le volet « danse internationale » de l'offre du festival montréalais. Sans qu'elles soient aussi fortes, la programmation du FTA a aussi des affinités avec celles du Festival d'Avignon (7 spectacles ou un par édition), du Festival d'Automne de Paris (7), de la Bâtie de Genève (6), du Wiener Festwochen (5) ou du défunt Spielzeit'Europa qu'organisait le Berliner Festspiele (5), pour ne nommer que ceux là.

Dans la perspective des réseaux de diffusion, ces communautés électives apparaissent significatives pour deux raisons. D'abord, parce qu'elles se concentrent autour de quelquesunes des principales têtes d'affiche du théâtre contemporain, elles soulignent l'intégration du FTA dans un espace international fondé sur des spectacles, des démarches et des esthétiques partagés. Ensuite, elles peuvent donner lieu, dans certaines circonstances, à des réseaux de coproduction dont le propre est de lancer de nouvelles œuvres au sein de cet espace international.

\section{Réseaux de coproduction}

Pour un festival comme le FTA, la coproduction signifie d'ordinaire un soutien financier offert à une compagnie de théâtre entre les premiers temps d'une création nouvelle et les dernières étapes d'une œuvre non encore diffusée. Parce qu'elle implique un investissement financier et un risque esthétique, la coproduction de spectacles, on l'aura compris, n'est pas à la portée de tous. ${ }^{17}$ La capacité de soutenir la création d'œuvres nouvelles, cependant, distingue les festivals 
coproducteurs des festivals diffuseurs, c'est-à-dire ceux qui sont à l'origine des œuvres et ceux qui se contentent de les relayer. Si ces derniers agissent du côté de la demande et tendent à être acheteur de spectacles, qu'ils soient ou non achevés, ${ }^{18}$ les premiers se situent davantage du côté de l'offre en soutenant en amont la création de nouveaux biens culturels à proposer.

En échange d'un soutien qui peut dans certains cas s'avérer décisif, un coproducteur comme le Festival TransAmériques, qui investit dans huit spectacles en moyenne par édition, retire un certain nombre d'avantages symboliques et stratégiques dont celui de pouvoir proposer-en "création mondiale " (Couturier, Io juin 2013) - quelques-uns des spectacles qu'il aura en partie financés. ${ }^{19}$ Tout aussi important est que la coproduction permet à un festival d'associer très étroitement son nom et sa réputation au devenir d'un artiste. Association étroite, en effet, puisque le nom du coproducteur est systématiquement inclus, à la manière d'un label d'origine, dans l'ensemble des documents papiers ou virtuels qui accompagnent un spectacle en tournée nationale ou internationale : matériel promotionnel, programme de soirée, site web, blogues, etc. Si ce genre d'informations passe d'ordinaire inaperçu aux yeux de la plupart des festivaliers, tel n'est vraisemblablement pas le cas pour la catégorie des professionnels de la scène à qui importe, justement, pareil « détail ». Dès lors, par le biais des coproductions auxquelles il participe, c'est aussi le nom et la réputation du FTA qui circulent dans de multiples réseaux de diffusion : ceux de Brassard, de Lepage, de Marleau ou de Mouawad, notamment, suivant les spectacles qu'il a coproduit.

Prenons un exemple devenu emblématique. Présenté en novembre 2000 à l'Espace libre de Montréal, fimmy, créature de rêve, le solo inaugural de Marie Brassard, est une version revue et augmentée d'un premier essai présenté en lever de rideau de Pension Vaudou, un spectacle dans lequel Brassard ne jouait pas elle-même. Présente dans la salle, Marie-Hélène Falcon est séduite par la performance et il est rapidement convenu que Brassard travaillera à augmenter cette première version en vue d'une présentation, au printemps suivant, dans le cadre du FTA (Guay).

Le spectacle est donc (re)créé à Montréal pour une série de six représentations données du 3 au 7 juin 200 I dans l'intimité de la salle Jean-Claude-Germain ( 75 fauteuils). Cette annéelà, fimmy était l'une de trois coproductions dans lesquelles le FTA se trouvait impliqué : une exception, en somme, parmi les I 8 spectacles qui composaient sa 9 ième édition. Le solo attire rapidement l'intérêt et les invitations de diffuseurs-programmateurs locaux et étrangers. D'invitation en invitation, entre juin 200I et novembre 2008, Fimmy aura été présenté dans trente-huit villes différentes - huit canadiennes et trente à l'étranger-réparties sur seize pays et trois continents. Le label FTA, à titre de coproducteur unique du spectacle, s'est donc intégré à la programmation de festivals ou de diffuseurs dont la répartition géographique est illustrée par la prochaine carte.

Manifestement, toute une série de transactions s'effectue ici, par l'intermédiaire de ce spectacle que le FTA a coproduit et que d'autres ont diffusé ; des alliances objectives dans lesquelles des festivals ou des diffuseurs étrangers cautionnent une décision artistique du FTA en l'adoptant pour leur propre évènement. De telles cooptations désignent non seulement le FTA comme un interlocuteur légitime au sein de cet espace, elles en font un festival qui, cessant d'être uniquement un relayeur de spectacles, contribue désormais au contenu des réseaux de diffusion auxquels il participe par ailleurs.

Une fois vu dans ces espaces internationaux de diffusion, un artiste québécois peut beau- 


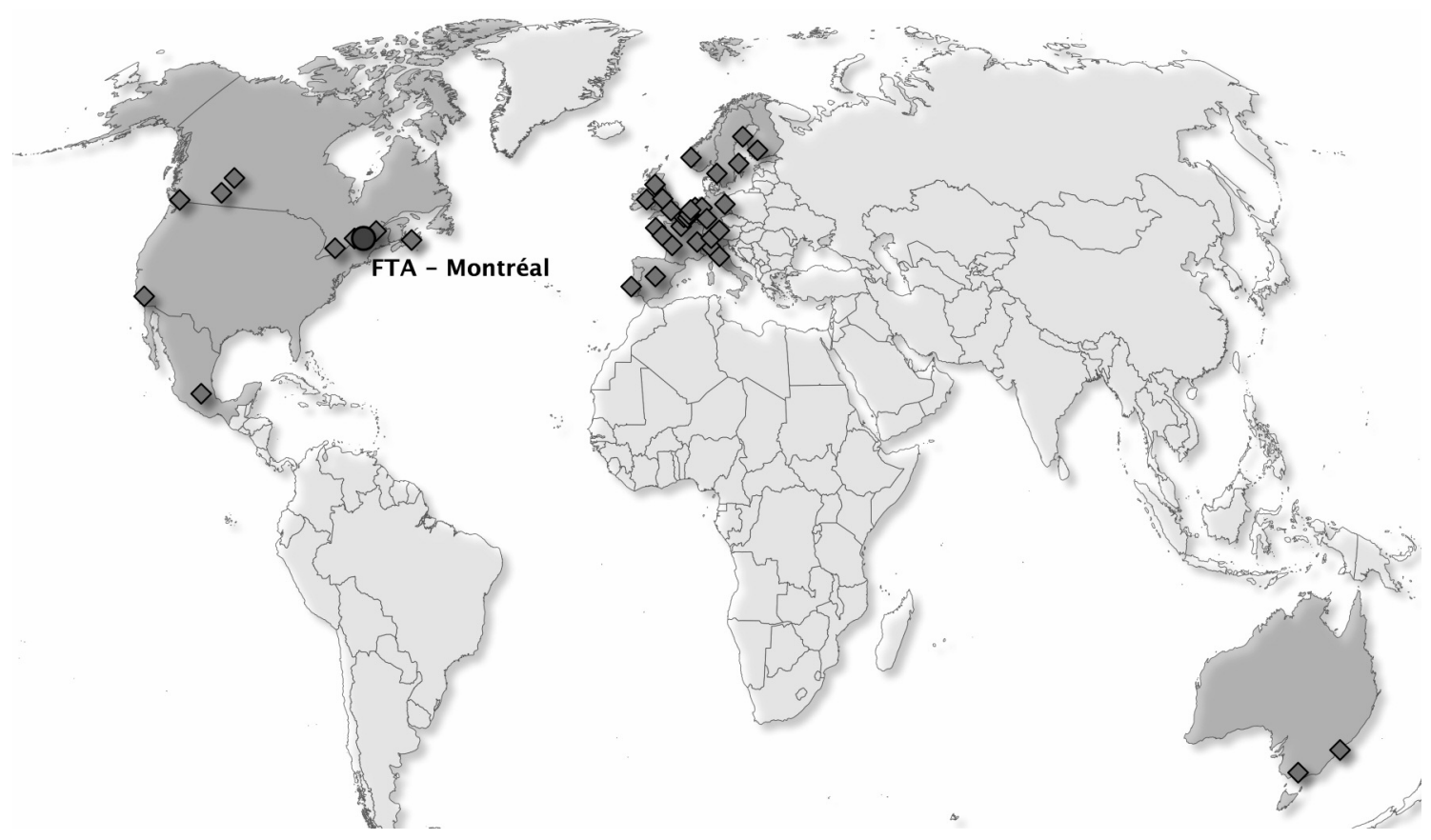

Réseau de diffusion de Jimmy, créature de rêve (Marie Brassard) (2001-2008;

FTA, coproducteur unique)

coup plus facilement trouver de nouveaux appuis pour un futur spectacle parmi les festivals et diffuseurs saisonniers qui l'ont accueilli. C'est le cas de Brassard qui, dans la foulée de l'intérêt suscité par fimmy, a pu poursuivre sa démarche avec La Noirceur (2003), Peepshow (2005), Linvisible (2008) ou Moi qui me parle à moi-même dans le futur (2OII) grâce au soutien continu du FTA, mais aussi à celui de nouveaux coproducteurs nationaux et internationaux, dont La Bâtie-Festival de Genève, le Spielzeit'Europa du Berliner Festspiele ou le Wiener Festwochen qui se sont joints à titre de coproducteurs une fois lancée sa carrière solo.

Par l'entremise des spectacles québécois qu'il coproduit avec d'autres festivals et diffuseurs saisonniers, le FTA noue ainsi des alliances passagères avec ses pairs à l'étranger, alliances dont l'une des finalités consiste à financer, par une mise en commun de capitaux nationaux et étrangers, la création d'un certain théâtre québécois. Vus en diachronie, les réseaux de coproductions du FTA forment ainsi la toile complexe des interlocuteurs du festival depuis sa refonte en 2007. Afin de donner une meilleure idée de ses ramifications, je ne propose plus bas (p. 3I2) que l'agrandissement de sa zone la plus dense, celle des réseaux de l'Europe continentale où circule le nom du FTA.

Entre 2007 et 2013, le festival a participé à quatre-vingt-trois coproductions, dont cinquante-cinq avec des compagnies de théâtre. Ce faisant, ses ressources ont été mises en commun avec celles de plus de soixante-dix festivals et autres diffuseurs à l'étranger, et une dizaine au Canada. Pour importantes que puissent paraître les ramifications internationales du FTA, l'étendue de son réseau de coproduction est en réalité inversement proportionnelle à la force des liens qui le constituent. On dira des liens entre coproducteurs qu'ils sont faibles lorsqu'ils sont très nombreux à être impliqués dans un même projet et que leur alliance ne se 


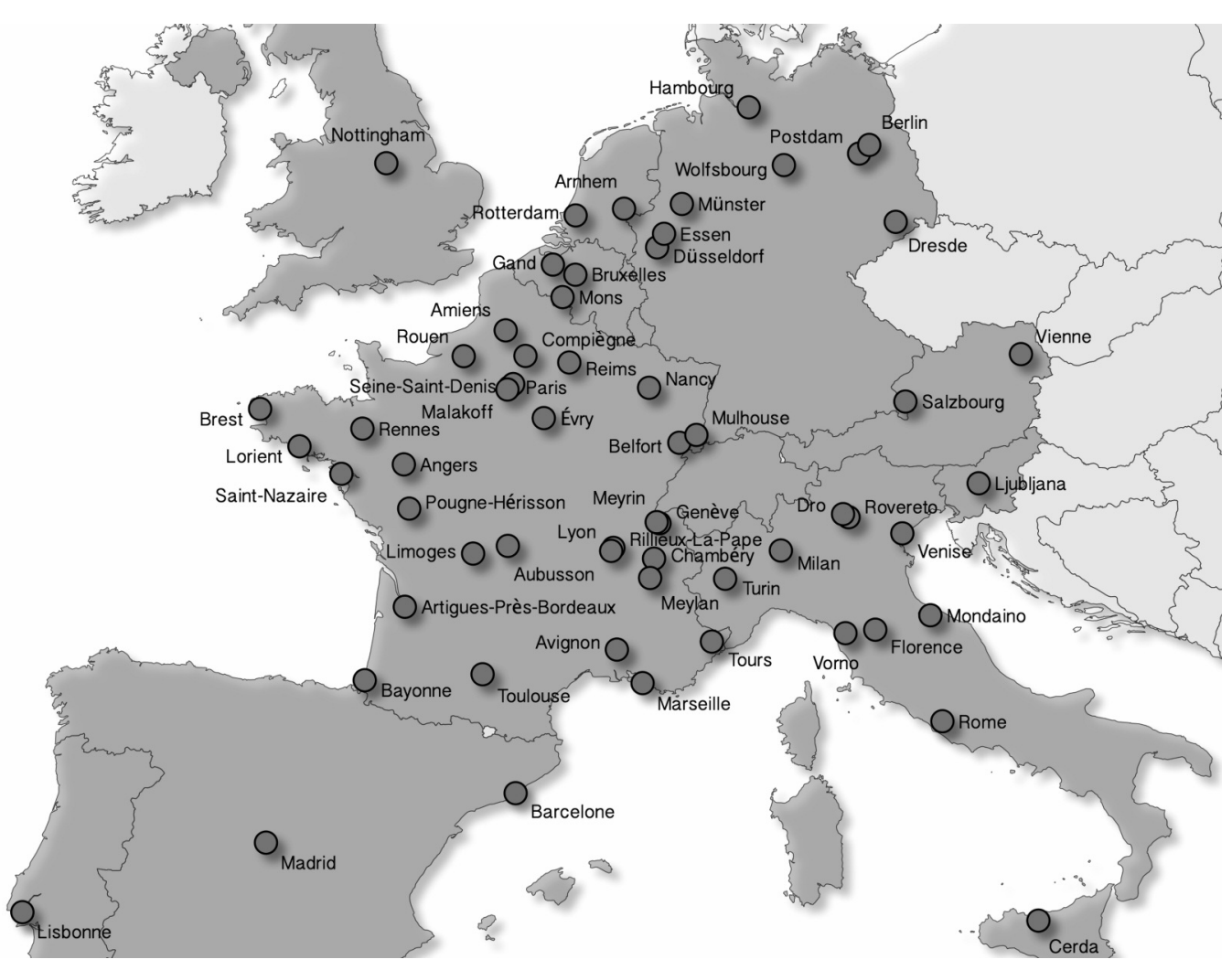

Festival TransAmériques (2007-2013) Réseaux de coproductions européens

renouvèle pas autour d'autres spectacles. Un exemple emblématique pourrait être Le sang des promesses de Wajdi Mouawad, trilogie reprise en 2009 au Festival d'Avignon dans une vaste coproduction à vingt-six, incluant le FTA. Dans pareil cas, le réseau de coproductions d'un spectacle s'apparente en fait à un réseau de diffusion.

Leur étendue donne néanmoins un exemple de la structure financière complexe sur laquelle repose aujourd'hui une part de l'économie festivalière, mais aussi celle du théâtre québécois de pointe qui en bénéficie. En tant que modèle économique, la coproduction apparaît ainsi comme un moyen de développer une tournée par souscription. Le spectacle y est en quelque sorte mis en prévente, sa pertinence garantie par le parcours et la démarche de l'artiste comme par le flair reconnu à ses coproducteurs.

\section{La coproduction internationale}

Le lien fort avec les partenaires étrangers, c'est un rôle plus déterminant dans la coproduction internationale qui serait susceptible de le générer. Mais lorsqu'il s'agit de créer un spectacle du Nature Theatre of Oklahoma, de La Ribot ou d'autres artistes étrangers, renommés ou non, force est d'admettre que le FTA n'est pas un interlocuteur pour ses pairs, sinon à titre de diffuseur potentiel des artistes que ces derniers soutiennent. 
Si l'on s'en tient à l'origine des artistes coproduits par le FTA, on constate que le champ d'action du festival se limite largement à la sphère nationale. Pour étendus qu'ils soient, ses réseaux de coproduction ne s'actualisent, pour l'essentiel, qu'à partir du moment où un artiste québécois y occupe la position centrale. Certes, cela ne l'empêche pas d'avoir une réelle présence à l'étranger, on l'a vu. Simplement, si le FTA est coproducteur d'un spectacle avec le Festival d'Avignon, Le Manège de Mons, le Cankarjev Dom de Ljubljana ou la Scène nationale de Petit-Quevilly Mont-Saint-Aignan, c'est surtout parce que Denis Marleau ou Wajdi Mouawad sont impliqués.

À ce jour, le festival n'a piloté que deux projets de coproductions internationales : un premier avec les New York City Players de Richard Maxwell (Neutral Hero, 20II) et un second avec la compagnie italienne Motus d'Enrico Casagrande et Daniela Nicolò (Nella Tempesta, 2013). Dans les deux cas, ces artistes avaient déjà présenté leur travail au public du festival montréalais qui l'avait accueilli très favorablement, faisant de la coproduction la suite logique d'une relation déjà amorcée.

Bien que rarissimes, de telles aventures sont pour le FTA l'occasion d'élargir ses réseaux au-delà de ceux qu'il a en partage avec quelques artistes du Québec. Tout comme Marie Brassard a des soutiens québécois et canadiens qui lui sont fidèles à différents degrés (FTA, Usine C, PuSh, Théâtre français du Centre National des Arts), une compagnie comme Motus possède des soutiens semblables en Italie, en France et ailleurs en Europe (Festival Delle Colline Torinesi, Théâtre National de Bretagne, etc.). Ce sont à de tels réseaux que peut se brancher le FTA en finançant une compagnie étrangère, réseaux que le festival n'aurait vraisemblablement pas pu investir en suivant le seul rayonnement international des artistes québécois qu'il soutient en danse et en théâtre. En retour, il n'est pas interdit de penser que ces nouveaux espaces puissent générer d'autres alliances et profiter aux artistes québécois que soutient par ailleurs le festival.

La coproduction internationale transforme ainsi un festival en interlocuteur potentiel au sein d'un espace qui, pour le coup, devient réellement supranational puisqu'en partie dégagé de l'obligation (politique, économique, symbolique) de soutenir uniquement les artistes issus du pays producteur. Or, le propre de l'internationalisation des réseaux étrangers de coproduction - des compagnies québécoises en dépendent-est aussi de mettre en commun une part du capital économique dévolu à la culture de pointe, capital qui, en Europe comme au Canada, provient largement des coffres publics. Par l'entremise des festivals et diffuseurs qui s'y adonnent, par toute une série de vases communicants dont on peut imaginer les ramifications, la coproduction internationale permet aussi de soutenir l'art du Québec grâce à des fonds étrangers (français, allemands, belges, anglais, etc.). Mais rarement assisteon à l'inverse.

Les raisons qui expliquent cette non-réciprocité des échanges sont complexes et multiples. Si la coproduction internationale n'est pas à proprement parler interdite par les programmes des trois conseils des arts qui soutiennent le FTA, elle n'est pas pour autant une pratique facile, ou même souhaitable en contexte canadien. Les distances, d'abord, ne font pas nécessairement du FTA un partenaire intéressant s'il demeure seul impliqué de son côté de l'Atlantique. Car la coproduction, en effet, est aussi une manière d'aider à développer une tournée et, pour des compagnies étrangères-notamment européennes-, se déplacer en Amérique peut ne pas être un choix viable si un seul arrêt est à prévoir. De même, la faible 
densité de la population sur le territoire canadien offre peu de partenaires au festival avec qui se lancer dans l'aventure internationale. La rareté des fonds disponibles explique aussi en partie cette implication limitée du FTA comme celle d'autres festivals canadiens. Puis, il y a le fait que la coproduction internationale rompt le lien entre l'origine nationale des fonds destinés à la production des spectacles et la nationalité des artistes qui en bénéficient. Dans le contexte nord-américain où cette culture de la réciprocité est inexistante, des voix se lèveraient sans doute si des fonds nationaux-déjà jugés insuffisants - en venaient à soutenir la production de nombreux spectacles étrangers.

Peu en importent les causes, l'une des conséquences majeures de cette non-réciprocité au sein du réseau est que le FTA n'est pas d'emblée perçu-pas plus d'ailleurs que ses vis-àvis québécois ou canadiens - comme un partenaire potentiel dans la coproduction des artistes (flamands, italiens, japonais, russes, chiliens, etc.) que soutiennent ses pairs étrangers. Coproduire à l'international apparaît dès lors comme la condition d'entrée à de nouveaux réseaux, ce qui constitue sans doute le plus grand défi de la prochaine équipe de direction artistique du Festival TransAmériques. ${ }^{20}$

\section{Notes}

I Le nombre de spectacles québécois ayant tourné à l'étranger est de 7 pour la décennie 1960, 30 pour la suivante et 54 pour la décennie 1980 . Entre 1990 et 2000,80 spectacles de théâtre québécois ont été joués hors du Canada. Ces chiffres proviennent pour l'essentiel d'une enquête menée auprès de 25 compagnies théâtrales du Québec, dont de nombreuses compagnies jeunes publics (Schryburt 1999).

2 Il importe ici de souligner qu'à la même période, les compagnies jeunes publics du Québec circulent aussi beaucoup à l'étranger, voire même davantage que leur homologues pour « adultes ». Pensons à des spectacles tels que Pleurer pour rire de Marcel Sabourin (Théâtre de la Marmaille [aujourd'hui Les Deux Mondes], I98I), Une lune entre deux maisons de Suzanne Lebeau (Le Carrousel, 1982) ou Mur-Mur de Robert Dion (DynamO Théâtre, 1987). Attendu les particularités de son rayonnement international (réseaux, instances de consécration, longévité des spectacles, etc.), l'étude de ce secteur dépasserait largement le cadre nécessairement limité de la présente analyse.

3 Coup de théâtre, au moment de terminer ce texte, Falcon annonçait sa décision de quitter la direc-

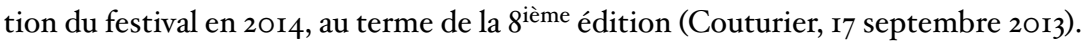

4 Pour un bilan critique du FTA, version Amériques et TransAmériques, je renverrai le lecteur à l'article de Gilbert David publié en 2009 dans la revue Spirale.

5 De 2I à 23 spectacles par édition, pour être exact. Seules les éditions 20 II et 20 I2 diffèrent avec 3I et 27 spectacles respectivement.

6 Les catégories « danse » et « théâtre » sont celles qu'emploie le festival dans ses programmes d'édition.

7 Entendre ici « saison théâtrale ", par opposition au modèle de "l'édition " qui est celui des festivals et qui suppose un tout autre rapport au temps.

8 Ces diffuseurs saisonniers sont aussi-pour certains d'entre eux-des organisateurs de festivals, ce qui souligne encore, si besoin était, les liens étroits qui unissent diffuseurs saisonniers et festivals dans les circuits mondialisés du théâtre contemporain.

9 Rencontres avec les artistes, mises en lecture, laboratoires, cocktails et autres évènements sociaux, 
débats, congrès, colloques, rencontres professionnelles, etc.

Io J'ajouterais l'adjectif "public " à cette définition afin de distinguer les festivals des autres métaévènements que sont les «showcases» largement tournés vers l'industrie et le public spécialisé des professionnels du spectacle (ex. : le Réseau Indépendant des Diffuseurs d'Évènements Artistiques Unis ou RIDEAU).

II Outre l'Usine C, on peut penser ici au Théâtre La Chapelle, dont le champ d'action international est beaucoup plus restreint.

I2 En anglais : «structure of relationships linking social actors ».

I3 Dans la terminologie de la sociologie des réseaux d'acteurs sociaux, ce public spécifique d'un festival pourrait être désigné comme le " cercle» (Degenne et Forsé 2006: 69-70) des professionnels.

I4 Ce chiffre exclut les « artistes » (218) et les « journalistes» (I28), deux catégories qui ouvrent à l'étude d'autres réseaux que ceux qui m'intéressent ici.

I5 Qui sont-ils? Quelles institutions représentent-ils? Pourquoi fréquentent-ils le festival?

I6 Ces réseaux de diffusion créent aussi des communautés électives transnationales fondées sur des expériences théâtrales partagées entre diverses catégories de spectateurs (amateurs, critiques, professionnels, chercheurs universitaires, etc.).

I7 Suivant les derniers chiffres disponibles (i.e. 20I2-20I3), le FTA a reçu 83I 500\$ du Conseil des arts et des lettres du Québec (Appendice au rapport annuel de gestion du Conseil des arts et des lettres $d u$ 2uébec), 300 ooo\$ de Patrimoine canadien (Patrimoine), 174 ooo\$ du Conseil des arts du Canada (Conseil) et I70 ooo\$ du Conseil des arts de Montréal (Rapport annuel 20I2, 20I3). De cette somme, qui exclut notamment les revenus autonomes du FTA, il m'est impossible de dire combien de fonds le festival consacre à la coproduction de spectacles.

I8 J'en veux pour preuve cette déclaration d'une candeur éclairante : «Dans le fascicule distribué aux spectateurs du Teatro Circo Price [de Madrid, lieu de la création de l'avant-dernier Lepage], on peut lire que la Round House de Londres a acheté Pique les yeux fermés : "Nous ne savons rien du contenu, mais nous savons que ce sera merveilleux", disait son directeur. Après tout, comme l'écrivait le quotidien espagnol $A B C$, "Lepage est le génie des nouvelles technologies" "(Robitaille 2OI2).

I9 Ces «créations mondiales » et autres variantes du genre (primeur ou exclusivité nord-américaine, par exemple) mettent en valeur l'antériorité ou l'exclusivité de l'offre d'un festival comme le FTA et agissent tels des marqueurs de distinction entre acteurs du champ international.

20 Au moment de mettre cet article sous presse, on apprenait que c'est Martin Faucher qui prendra la relève de Marie-Hélène Falcon à la direction du Festival TransAmériques. Lambition de joindre la ligue des coproducteurs à l'international figure en bonne place parmi les priorités identifiées par Faucher au lendemain de sa nomination : «Le festival coproduit des créations chaque année, comme Solitudes solo de Daniel Léveillé ou Trieste de Marie Brassard, mais surtout sur la scène nationale. "Il faut que Montréal se positionne comme partenaire majeur dans la coproduction, [. . . et quỉl y ait une réciprocité [avec la scène internationale]. C'est important que les subventionneurs le comprennent."»(Doyon 2014)

\section{Ouvrages cités}

Charlebois, Marie-Ève (source). "Carrefour International de Théâtre. Bilan de la $\mathrm{I}_{4}^{\mathrm{e}}$ édition. » Communiqué officiel du Carrefour International de Théâtre de Québec, I3 juin 2013. Web. 28 août 2013 .

Conseil des arts du Canada. S.p., s.d. Web. 30 déc. 2013. 
Conseil des arts et des lettres du Québec. Appendice au rapport annuel de gestion du Conseil des arts et des lettres $d u$ 2uébec. S.p., sept. 2013. Web. 30 déc. 2013.

Conseil des arts de Montréal. Rapport annuel 2012. S.p., 2013. Web. 30 déc. 2013.

Couturier, Hugo (source). « Marie-Hélène Falcon cède les rênes du FTA. » Communiqué officiel du Festival TransAmériques, 17 sept. 2013. Web. I7 sept. 2013.

—. «Le Septième Festival TransAmériques : les spectateur au cœur de l'action. » Communiqué officiel du Festival TransAmériques, Io juin 2013. Web. 28 août 2013.

David, Gilbert. «Lavènement du Festival de Théâtre des Amériques (1985), ou l'inscription d'un régime esthétique sans frontières. »Spirale 228 (2009) : I26-27. Imprimé.

Degenne, Alain et Michel Forsé. Les Réseaux sociaux. Paris : Armand Collin, coll. «U», 2004. Imprimé.

Doyon, Frédérique. «Une succession sous le signe de la continuité au FTA. » Le Devoir II fév. 2014.

Ferguson, Alex Lazaridis. «Symbolic Capital and Relationships of Flow : Canada, Europe, and the International Performing Arts Festival Circuit. » Theatre Research in Canada / Recherches théatrales au Canada 34.2 (2013) : 97-124. Imprimé.

Festival d'Avignon. S.p., s.d. Web. I8 août 2013.

Guay, Hervé. «Sur les scènes du monde-Opter pour l'aventure. » Le Devoir 6 oct. 2007. Imprimé.

Lacroix, Michel. «Littérature, analyse de réseaux et centralité : esquisse d'une théorisation du lien social concret en littérature. » Recherches sociographiques 44.3 (2003) : 475-97. Imprimé.

Lemieux, Vincent. Les Réseaux d'acteurs sociaux. Paris : Presses Universitaires de France, coll. «Sociologie », I999. Imprimé.

Marsden, Peter V. « Social Networks. » Encyclopedia of Sociology. Dir. Edgar F. Borgatta et Rhonda J. V. Montgomery. New York : Macmillan, 2000. 2727-35. Imprimé.

Mesure, Sylvie et Patrick Savidan, dir. Le Dictionnaire des sciences humaines. Paris : Presses

Universitaires de France, coll. «Quadrige », 2006. Imprimé.

Patrimoine canadien. S.p., s.d. Web. 30 déc. 2013.

PuSh. Annual Report. PuSh. Cross the Line, 2013. Web. 28 août 2013.

Robitaille, Louis-Bernard, «Pique de Robert Lepage : une œuvre éblouissante. » La Presse 15 mai 2012. Imprimé.

Schoenmakers, Henri. « Festivals, Theatrical Events and Communicative Interactions » Festivalising! Theatrical Events, Politics and Culture. Dir. Temple Hauptfleisch, Shulamith Lev-Aladgem, Jacqueline Martin, Willmar Sauter et Henri Schoenmakers. Amsterdam / New York, Rodopi, tome 3 de la série "Themes in Theatre. Collective Approaches to Theatre and Performance ", 2007. 27-37. Imprimé.

Schryburt, Sylvain. Repère signalétique des productions de compagnies théâtrales du 2uébec jouées à l'étranger. Premier État, cahier de recherche $\mathrm{n}^{\circ}$ I4. Montréal : Centre d'études québécoises (CÉTUQ), I999. Imprimé.

—. "Quebec Theatre: New Dynamics between the Local and the International. " 2uebec 2uestions: 2uebec Studies for the Twenty-First Century. Dir. Stéphan Gervais, Christopher Kirkey et Jarrett Rudy. Oxford: Oxford University Press, 20IO. 443-55. Imprimé.

—. «Les festivals de théâtre : esquisse d'une sociologie du champ théâtral international. »Une histoire des festivals. $X X^{e}-X X I^{e}$ siècle. Dir. Anaïs Fléchet, Pascale Goetschel, Patricia Hidiroglou, Sophie Jacotot, Caroline Moine et Julie Verlaine. Paris : Publications de la Sorbonne, coll. "Histoire contemporaine ", 2013. 139-150. Imprimé. 